University of Nebraska - Lincoln

DigitalCommons@University of Nebraska - Lincoln

$11-2013$

\title{
Harmonic generation spectroscopy with a two-colour laser field having orthogonal linear polarizations
}

\author{
T. S. Sarantseva \\ Voronezh State University, sartan86@mail.ru
}

M. V. Frolov

Voronezh State University, Russia, frolov@phys.vsu.ru

N. L. Manakov

Voronezh State University, manakov@phys.vsu.ru

M. Yu. Ivanov

Voronezh State University

Anthony F. Starace

University of Nebraska-Lincoln, astarace1@unl.edu

Follow this and additional works at: https://digitalcommons.unl.edu/physicsstarace

Part of the Atomic, Molecular and Optical Physics Commons, Elementary Particles and Fields and String Theory Commons, and the Plasma and Beam Physics Commons

Sarantseva, T. S.; Frolov, M. V.; Manakov, N. L.; Ivanov, M. Yu.; and Starace, Anthony F., "Harmonic generation spectroscopy with a two-colour laser field having orthogonal linear polarizations" (2013). Anthony F. Starace Publications. 203.

https://digitalcommons.unl.edu/physicsstarace/203

This Article is brought to you for free and open access by the Research Papers in Physics and Astronomy at DigitalCommons@University of Nebraska - Lincoln. It has been accepted for inclusion in Anthony F. Starace Publications by an authorized administrator of DigitalCommons@University of Nebraska - Lincoln. 


\title{
Harmonic generation spectroscopy with a two-colour laser field having orthogonal linear polarizations
}

\author{
T S Sarantseva ${ }^{1}$, M V Frolov ${ }^{1}$, N L Manakov ${ }^{1}$, M Yu Ivanov ${ }^{1,2}$ \\ and Anthony F Starace ${ }^{3}$ \\ ${ }^{1}$ Physics Department, Voronezh State University, 394006 Voronezh, Russia \\ ${ }^{2}$ Max-Born Institute, Max-Born-Strasse 2A, D-12489 Berlin, Germany \\ ${ }^{3}$ Department of Physics and Astronomy, The University of Nebraska, Lincoln, NE 68588-0111, USA \\ E-mail: sartan86@mail.ru
}

Received 3 October 2013, in final form 18 October 2013

Published 20 November 2013

Online at stacks.iop.org/JPhysB/46/231001

\begin{abstract}
The interpretation of many high-order harmonic generation (HHG) experiments is based on the assumption that the HHG yield of an atom can be factorized into (i) a laser-dependent 'electron wave packet' with rather simple properties, including a nearly universal shape, and (ii) an atomic photorecombination cross section. We show that this factorization is restricted to linearly polarized laser fields and fails in two-colour laser fields with orthogonal polarizations. At the same time, we show how two-colour HHG spectroscopy using orthogonally polarized intense fundamental and relatively weak second harmonic fields makes a complete experiment possible that enables the retrieval of the angle-resolved photorecombination cross sections for atomic $\mathrm{p}$ states.
\end{abstract}

(Some figures may appear in colour only in the online journal)

In addition to its important technological applications, such as the production of attosecond pulses, high-order harmonic generation (HHG) is now widely used in spectroscopic applications, allowing one to image atomic and molecular dynamics [1, 2]. Most experiments on HHG spectroscopy have been performed with a linearly polarized laser field. Their interpretation is often based on the idea that the frequencydomain HHG yield can be factorized in terms of an electron wave packet (EWP) that depends on the parameters of the strong laser field driving the harmonic emission and the (field-free) photorecombination cross section (PRCS) of the atomic or molecular target [3]. This assumption has been successfully used to retrieve the PRCS (or, equivalently, the photoionization cross section, which can be related to the PRCS using the principle of detailed balance) from measured HHG spectra (see, e.g., [2]). For a linearly polarized field, this factorization was proposed phenomenologically, based on numerical solutions of the time-dependent Schrödinger equation [3, 4]. It has now been justified theoretically for a monochromatic field [5, 6], for a short laser pulse [7-9], and for a two-colour field with collinearly polarized components [10].

While HHG spectroscopy can measure distinct features in the photorecombination of an electron to the outer p-subshells of rare gas atoms, such as the Cooper minimum in $\operatorname{Ar}[11,12]$ and the giant dipole resonance in Xe [2], HHG measurements for linear polarization are fundamentally limited. Specifically, they can only give access to the energy dependence of the differential PRCS $\sigma(E, \alpha)$ (where $\alpha$ is the angle between the photon polarization axis and the recombining electron's momentum). This is because the factorized result for HHG rates involves the PRCS $\sigma(E, \alpha)$ only for $\alpha=0$, while its angular dependence is described by the asymmetry parameter $\beta[13]$ :

$$
\sigma(E, \alpha)=\frac{\sigma_{0}}{4 \pi}\left(1+\beta \frac{3 \cos ^{2} \alpha-1}{2}\right),
$$


where $\sigma_{0}$ is the total PRCS. Thus, for a given electron energy $E$, 'one-dimensional' HHG spectroscopy with a linearly polarized field, provides only a single atomic parameter and does not allow one to retrieve the angular dependence of $\sigma(E, \alpha)$. Adding a phase-locked field polarized orthogonally to the fundamental driving field turns one-dimensional HHG spectroscopy into multi-dimensional spectroscopy [14-17] and provides access to more detailed information about the atomic and molecular response to the laser field, including the contributions of multiple ionic states and ionization timedelays. As shown in [16], the parameters $\sigma_{0}$ and $\beta$ can be retrieved from HHG spectra in an elliptically polarized field with small ellipticity by means of polarization measurements, i.e. by measuring the yield of harmonics linearly polarized along the major and minor axes of the polarization ellipse.

Here we suggest an alternative way to retrieve both $\sigma_{0}$ and $\beta$ from HHG spectra, avoiding polarization measurements, by using a two-colour field with orthogonally polarized components and measuring yields of odd and even harmonics. For an initial $\mathrm{p}$ state, we show that two degenerate orbitals aligned along the fundamental and second harmonic fields must be included in the description of the HHG signal, irrespective of the strength of the second harmonic field. Consequently, the factorization of the HHG yield is not possible in this case. However, our results provide a means to completely determine $\sigma(E, \alpha)$ using two-colour HHG spectroscopy.

Consider a model system of an electron in a shortrange potential $U(r)$ having a single bound state, $\psi_{\kappa l m}(\mathbf{r})=$ $\varphi_{\kappa l}(r) Y_{l, m}(\hat{\mathbf{r}})$, with energy $E_{0}=-\hbar^{2} \kappa^{2} /(2 m)$, angular momentum $l$ and its projection $m$. This model permits an exact solution of the HHG problem within the framework of time-dependent effective range (TDER) theory [18], which combines the quasistationary quasienergy state approach [19] (for an exact account of the electron interaction with a strong laser field) with effective range theory [20] (for the nonperturbative account of the electron interaction with the potential $U(r)$ in terms of the scattering phase $\delta_{l}(E)$ in the $l$-wave channel). This approach for describing HHG by a linearly polarized field has been presented in detail in [21] (for a monochromatic field) and [10] (for a two-colour field with parallel linear polarizations) and was generalized to the case of elliptic polarization in [16]. We extend it here to the case of a two-colour field with orthogonal polarizations, whose electric vector is

$$
\mathbf{F}(t)=\hat{\mathbf{x}} F_{1} \cos (\omega t)+\hat{\mathbf{y}} F_{2} \cos (2 \omega t+\phi),
$$

where $F_{1}$ and $F_{2}$ are the amplitudes of the fundamental field of frequency $\omega$ and its second harmonic, and where $\phi$ is the phase shift between the two components.

Since the angular momentum projection $m$ is not conserved in the field $\mathbf{F}(t)$, it is convenient to use a superposition of degenerate field-free substates $\psi_{\kappa l m}(\mathbf{r})$ with different $m$ (where we assume the quantization axis $z$ is directed along the propagation direction of $\mathbf{F}(t))$ for the initial bound state. For an initial s $(l=0)$ or p $(l=1)$ state, we use the same superpositions as for the case of elliptic polarization [16]:

$$
\begin{aligned}
\psi_{\kappa l q}(\mathbf{r}) & =\varphi_{\kappa l}(r) f_{l, q}(\hat{\mathbf{r}}), \quad f_{l, 0}(\hat{\mathbf{r}})=Y_{l, 0}(\hat{\mathbf{r}}), \\
f_{1, \pm 1}(\hat{\mathbf{r}}) & =\left[Y_{1,1}(\hat{\mathbf{r}}) \pm Y_{1,-1}(\hat{\mathbf{r}})\right] / \sqrt{2},
\end{aligned}
$$

where $\varphi_{\kappa l}(r)=-\mathrm{i}^{l} \kappa^{3 / 2} C_{\kappa l} h_{l}^{(1)}(\mathrm{i} \kappa r)$ is the radial wave function in TDER theory [18], $h_{l}^{(1)}(x)$ is the spherical Hankel function of the first kind and $C_{\kappa l}$ is the asymptotic coefficient. $C_{\kappa l}$ and $\kappa$ are parameters of the problem. Note that the superpositions $f_{1, q}(\hat{\mathbf{r}})$ define substates $\psi_{q} \equiv \psi_{\kappa l q}(\mathbf{r})$ oriented along the three coordinate basis vectors: $\hat{\mathbf{x}} \| \mathbf{F}_{1}(q=-1)$, $\hat{\mathbf{y}} \| \mathbf{F}_{2}(q=1)$ and $\hat{\mathbf{z}}(q=0)$.

In the low-frequency (quasiclassical) approximation [21], the exact TDER result for the HHG amplitude reduces to a onedimensional integral similar to that for an elliptically polarized field [16], so that the harmonic rate $\mathcal{R}_{l, q}(N)(l=0,1)$ for the $N$ th harmonic with energy $E_{\Omega}=N \hbar \omega$ is given by

$$
\mathcal{R}_{l, q}(N)=\frac{(N \omega)^{3}}{2 \pi \hbar c^{3}(2 l+1)}\left|\mathbf{d}_{l, q}^{(N)}\right|^{2},
$$

where the $N$ th Fourier coefficient $\mathbf{d}_{l, q} \equiv \mathbf{d}_{l, q}^{(N)}$ of the fieldinduced dipole moment has the same form as in [16]:

$$
\begin{aligned}
\mathbf{d}_{l, q} & =\mathrm{e} \frac{2 C_{\kappa l}(\kappa a)^{1 / 2}}{\mathrm{i} T} \int_{T / 2}^{T} f_{l, q}\left(\hat{\mathbf{K}}_{i}(t)\right) \sqrt{\frac{\hbar \omega_{\mathrm{at}}^{2}}{S_{t_{i}, t_{i}}^{\prime \prime}}} \\
& \times \frac{\mathrm{e}^{-\mathrm{i}\left[S\left(t, t_{i}\right)-E t+E_{0} t_{i}\right] / \hbar}}{\left[v_{\mathrm{at}}\left(t-t_{i}\right)\right]^{3 / 2}}\left\langle\psi_{\kappa l q}|\mathbf{r}| \psi_{\mathbf{K}(t)}\right\rangle \mathrm{d} t, \quad T=\frac{2 \pi}{\omega},
\end{aligned}
$$

where in terms of the vector potential $\mathbf{A}(t)$ of $\mathbf{F}(t)$ we have

$$
\begin{aligned}
& \mathbf{A}(t)=-\frac{c}{\omega}\left[\hat{\mathbf{x}} F_{1} \sin \omega t-\hat{\mathbf{y}} \frac{F_{2}}{2} \sin (2 \omega t+\phi)\right], \\
& S\left(t, t_{i}\right)=\int_{t_{i}}^{t} \mathbf{K}^{2}\left(t^{\prime} ; t, t_{i}\right) /(2 m) \mathrm{d} t^{\prime}, \\
& \mathbf{K}\left(t^{\prime} ; t, t_{i}\right)=\frac{|e|}{c}\left[\mathbf{A}\left(t^{\prime}\right)-\frac{\int_{t_{i}}^{t} \mathbf{A}(\tau) \mathrm{d} \tau}{t-t_{i}}\right],
\end{aligned}
$$

where $E=E_{\Omega}-\left|E_{0}\right|, a=\hbar^{2} /\left(m e^{2}\right), v_{\text {at }}=e^{2} / \hbar, \omega_{\text {at }}=$ $v_{\text {at }} / a$ and $\mathbf{K}_{i}(t) \equiv \mathbf{K}\left(t_{i} ; t, t_{i}\right)$ and $\mathbf{K}(t) \equiv \mathbf{K}\left(t ; t, t_{i}\right)$, where $\mathbf{K}\left(t^{\prime} ; t, t_{i}\right)$ is the instantaneous (at time $\left.t^{\prime}\right)$ classical momentum of an electron that moves along a closed trajectory in the field $\mathbf{F}(t)$ starting at the time of ionization $t_{i}$ and returning at time $t$. The wave function $\psi_{\mathbf{K}(t)}(\mathbf{r})$ is a field-free scattering state of an electron with 'momentum' $\mathbf{K}(t)$ in the TDER theory [6]. For a given time $t$, the time $t_{i}=t_{i}(t)$ in (5) is determined as that root of the energy conservation equation applicable at the moment of ionization,

$$
\mathbf{K}^{2}\left(t_{i} ; t, t_{i}\right) /(2 m)=-\left|E_{0}\right|,
$$

which has a positive imaginary part and the smallest real part [6]. Note that the momentum $\mathbf{K}_{i}(t)$ lies in the $X Y$-plane, so that $f_{1,0}\left(\hat{\mathbf{K}}_{i}(t)\right)=0$ and HHG for the substate $\psi_{0}(\mathbf{r})$ is strongly suppressed and vanishes in the approximation (5).

The dipole moment (5) can be expressed in terms of its components as

$$
\mathbf{d}_{l, q}=d_{l, q}^{(\mathrm{o})} \hat{\mathbf{x}}+d_{l, q}^{(\mathrm{e})} \hat{\mathbf{y}},
$$

where $d_{l, q}^{(\mathrm{o})}\left(d_{l, q}^{(\mathrm{e})}\right)$ vanishes for even (odd) harmonics. Equation (7) shows that odd harmonics are linearly polarized along the direction $\hat{\mathbf{x}}$ of the fundamental field polarization, while even harmonics are linearly polarized along the direction $\hat{\mathbf{y}}$ of the second harmonic polarization, in agreement with results in [22] (see also [14]). In figure 1 we present 2D maps of the partial harmonic rates (4) of odd and even harmonics 


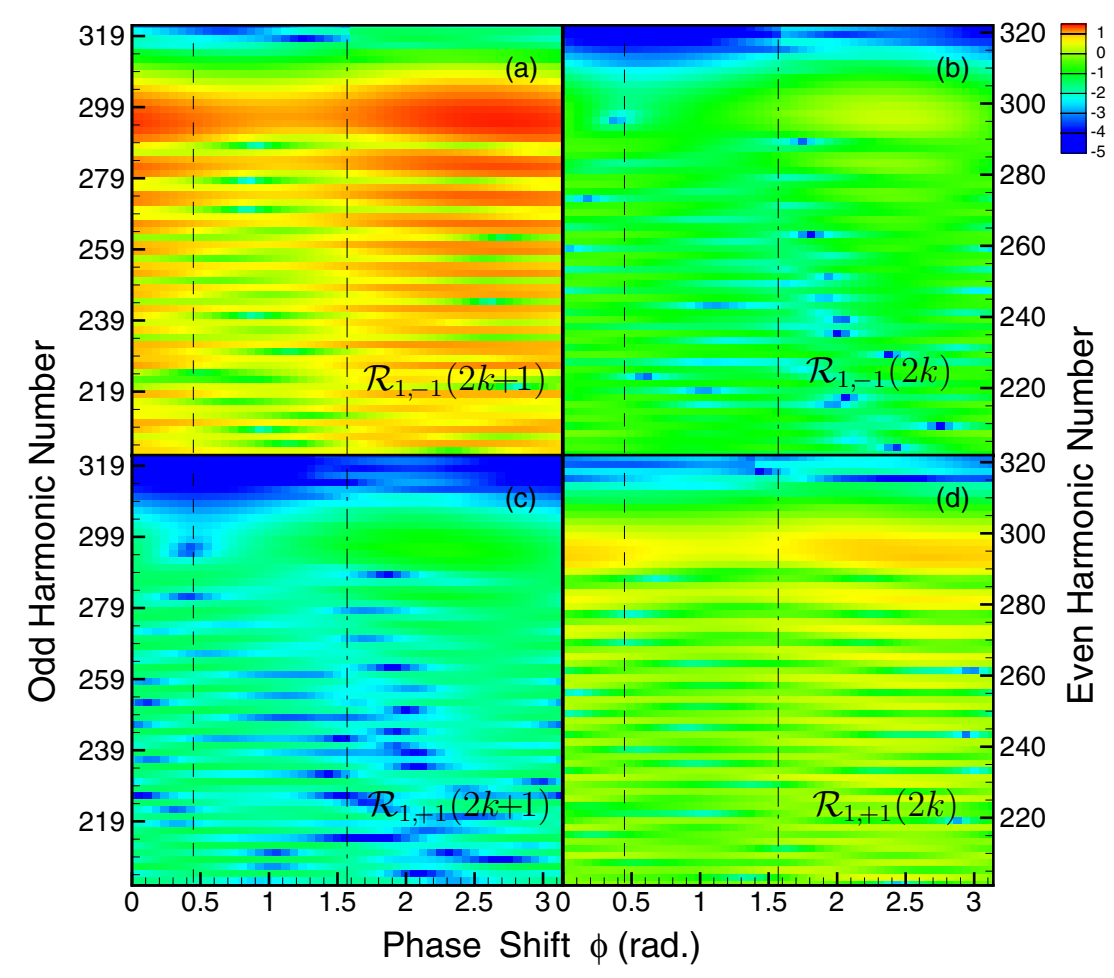

Figure 1. 2D map of the rates $\mathcal{R}_{1, q}(N)$ for odd ((a) and (c)) and even ((b) and (d)) harmonics as a function of the harmonic number and phase shift $\phi$ obtained using (4) and (5) for a bound p state with energy $E_{0}=-12.13 \mathrm{eV}$ (as for Xe). (a) and (b): results for the substate $\psi_{-1}$; (c) and (d): results for the substate $\psi_{+1}$. Intensities of the fundamental and second harmonics are $I_{1}=2 \times 10^{14} \mathrm{~W} \mathrm{~cm}^{-2}$ and $I_{2}=1.25 \times$

$10^{13} \mathrm{~W} \mathrm{~cm}^{-2}$ and the fundamental wavelength is $\lambda=1.8 \mu \mathrm{m}$. Dashed and dot-dashed vertical lines mark the results for $\phi=\pi / 7$ and $\pi / 2$.

for substates $\psi_{ \pm 1}$ as functions of the harmonic number and the phase shift $\phi$ obtained by numerical evaluations of the integral in (5). (For the explicit dependence of the recombination matrix element $\left\langle\psi_{\kappa l q}|\mathbf{r}| \psi_{\mathbf{K}(t)}\right\rangle$ in (5) on the vector $\mathbf{K}(t)$ see [16].) We note a number of qualitative features near the cutoff of HHG spectra for a $\mathrm{p}$ state:

(i) HHG spectra for both substates $\psi_{ \pm 1}$ contain even and odd harmonics, but the ratio of even and odd harmonic rates depends on the symmetry of $\psi_{q}$ : for $\psi_{-1}$ (oriented along the $x$ axis), the odd harmonics (polarized along $\hat{\mathbf{x}}$ ) are much more intense than even harmonics (polarized along $\hat{\mathbf{y}}$ ). (This feature holds also for an initial s state.) However, for the substate $\psi_{+1}$ (oriented along $\hat{\mathbf{y}}$ and providing the dominant contribution to the even harmonic signal) the opposite result holds: the even harmonic signal is much stronger than that for odd harmonics. These results follow from the fact that in photorecombination to an aligned $p$ state, the emission of a photon linearly polarized along the alignment direction is more probable than emission of a photon linearly polarized in the orthogonal direction.

(ii) The partial harmonic rates $\mathcal{R}_{1, q}(N)$ are sensitive to the phase shift $\phi$. Moreover, the phase dependence is most pronounced for the suppressed components of the harmonic spectrum (even harmonics for $\psi_{-1}$ and odd harmonics for $\psi_{+1}$ ). Whereas for the strong components only the magnitude of the harmonic yield depends on $\phi$, for the suppressed components both the shape of the plateau and the cutoff position are sensitive to the phase shift. That is because the dominant components of the HHG rate are proportional to the $x$-component of the returning electron momentum, which for the weak second harmonic field is not sensitive to the phase $\phi$. The suppressed components are proportional to the $y$ component of the momentum, which is highly sensitive to the phase $\phi$.

(iii) The spectra for both odd and even harmonics are qualitatively different for $\phi<\pi / 2$ and $\phi>\pi / 2$. For a time-delayed second harmonic field $(\phi<\pi / 2)$, the harmonic yields in figure 1 are suppressed compared to those for $\phi>\pi / 2$. Our analysis shows that this behaviour of the partial HHG yields originates from increased tunnelling ionization rates of the bound electron (described by the factor $\mathcal{T}$ in equation (13) below) for $\phi>\pi / 2$ as compared to those for $\phi<\pi / 2$.

The integral in (5) can be evaluated for a weak secondharmonic field, $F_{2} \ll F_{1}$, and an intense fundamental field, assuming a small Keldysh parameter, $\gamma=\hbar \omega \kappa /\left(|e| F_{1}\right) \ll 1$. Under these conditions, one can use the saddle-point method, in which case the main contribution to the integral in (5) comes from the saddle-points $t_{r}$ given by the equation

$$
\mathbf{K}^{2}\left(t_{r} ; t_{r}, t_{i}\right) /(2 m)=E .
$$

The system of equations (6) and (8) determines the set of solutions $\left\{t_{i}, t_{r}\right\}$ corresponding to closed electron trajectories. For the near-cutoff region of the HHG spectrum, only two ('short' and 'long') trajectories contribute to the HHG yield. Furthermore, at the cutoff these two trajectories merge to become the extremal one. To obtain the set of extremal times $\left\{t_{i}, t_{r}\right\}$, we first solve the classical equations for the times $\left\{t_{i}^{\mathrm{cl}}, t_{r}^{\mathrm{cl}}\right\}$ corresponding to the closed classical electron 
trajectory with the maximum classical energy $\mathcal{E}_{\max } \equiv$ $\mathbf{K}^{2}\left(t_{i}^{\mathrm{cl}} ; t_{i}^{\mathrm{cl}}, t_{r}^{\mathrm{cl}}\right) /(2 m)$ at the moment of return (see [16]):

$$
\begin{gathered}
\mathbf{K}\left(t_{i}^{\mathrm{cl}} ; t_{r}^{\mathrm{cl}}, t_{i}^{\mathrm{cl}}\right) \cdot \mathbf{K}\left(t_{r}^{\mathrm{cl}} ; t_{r}^{\mathrm{cl}}, t_{i}^{\mathrm{cl}}\right)=0, \\
\mathbf{K}\left(t_{r}^{\mathrm{cl}} ; t_{r}^{\mathrm{cl}}, t_{i}^{\mathrm{cl}}\right) \cdot \frac{\partial \mathbf{K}\left(t_{r}^{\mathrm{cl}} ; t_{r}^{\mathrm{cl}}, t_{i}^{\mathrm{cl}}\right)}{\partial t_{r}^{\mathrm{cl}}}=0 .
\end{gathered}
$$

Using the solution of the system (9), (10) as the zeroorder iteration of the system (6), (8), one can find the first-order quantum corrections to the classical times from the next iteration of the system (6), (8). We introduce dimensionless definitions for the laser field, $\mathbf{f}(t)=\mathbf{F}(t) / F_{1}$, the electron's momenta $\mathbf{q}\left(t, t_{i}\right)=\mathbf{K}\left(t_{i} ; t, t_{i}\right) /(\hbar \kappa)$ and $\mathbf{p}\left(t, t_{i}\right)=\mathbf{K}\left(t ; t, t_{i}\right) /(\hbar \kappa)$ and the time $\tau=\omega t$. Using these definitions and the notations $\mathbf{q}_{\mathrm{cl}} \equiv \mathbf{q}\left(t_{r}^{\mathrm{cl}}, t_{i}^{\mathrm{cl}}\right), \tau_{\mathrm{cl}}=\tau_{r}^{\mathrm{cl}}-\tau_{i}^{\mathrm{cl}}$ and $\gamma_{\tau}=\gamma / \tau_{\mathrm{cl}}=t_{\text {tun }} / t_{\mathrm{cl}}$ (where $t_{\text {tun }}=|e| F_{1} /(\hbar \kappa)$ is the Keldysh tunnelling time and $t_{\mathrm{cl}}=\tau_{\mathrm{cl}} / \omega$ is the electron's classical travel time in the continuum), we find for the first-order quantum corrections:

$$
\begin{aligned}
& \tau_{r} \approx \tau_{r}^{\mathrm{cl}}, \quad \tau_{i} \approx \tau_{i}^{\mathrm{cl}}+\gamma\left(\Delta_{\mathrm{re}}+\mathrm{i} \Delta_{\mathrm{im}}\right), \\
& \Delta_{\mathrm{re}}=\frac{\mathbf{q}_{\mathrm{cl}}}{\alpha} \cdot\left[\mathbf{f}\left(t_{i}^{\mathrm{cl}}\right)-\gamma_{\tau} \mathbf{q}_{\mathrm{cl}}\right], \\
& \Delta_{\mathrm{im}}=\sqrt{\frac{1+\mathbf{q}_{\mathrm{cl}}^{2}}{\alpha}-\Delta_{\mathrm{re}}^{2},} \\
& \alpha=\mathbf{f}^{2}\left(t_{i}^{\mathrm{cl}}\right)-\gamma_{\tau} \mathbf{q}_{\mathrm{cl}} \cdot\left[\tau_{\mathrm{cl}} \dot{\mathbf{f}}\left(t_{i}^{\mathrm{cl}}\right)-3 \mathbf{f}\left(t_{i}^{\mathrm{cl}}\right)-3 \gamma_{\tau} \mathbf{q}_{\mathrm{cl}}\right] .
\end{aligned}
$$

The saddle-point evaluation of the integral in (5) using equation (11) (cf the similar evaluation in [16]) leads to the final result for the (two-dimensional) vector $\mathbf{d}_{l, q}=\left(d_{x}^{(l, q)}, d_{y}^{(l, q)}\right) \equiv$ $\left(d_{l, q}^{(\mathrm{o})}, d_{l, q}^{(\mathrm{e})}\right)$ in (7) involving scalar, vector and matrix factors:

$$
\mathbf{d}_{l, q}=e \mathcal{T} \chi^{(l, q)} \hat{\mathcal{S}}_{l, q} .
$$

The factor $\mathcal{T}$ is a scalar describing the tunnelling of the electron from an initial bound state $\psi_{\kappa l q}(\mathbf{r})$ at the time $t=t_{i}^{\mathrm{cl}}$ :

$$
\mathcal{T}=\frac{\gamma \Delta_{\mathrm{im}}}{\pi} \sqrt{\frac{\Gamma_{\mathrm{st}}(\tilde{F})}{(2 l+1) \kappa v_{\mathrm{at}}}}, \quad \tilde{F} \equiv \frac{F_{1}}{\alpha \Delta_{\mathrm{im}}^{3}},
$$

where $\Gamma_{\text {st }}(\tilde{F})$ is the tunnelling detachment rate for a state $\psi_{\kappa l m=0}(\mathbf{r})$ in a static electric field of strength $\tilde{F} \hat{\mathbf{z}}[16,23]$. (Since $\Gamma_{\text {st }}(F) \propto(2 l+1)$ [23], the factor $\mathcal{T}$ is the same for both an s state and substates $\psi_{ \pm 1}$ of the p state.)

The factor $\chi^{(l, q)}$ in $(12)$ is a vector, $\chi^{(l, q)}=\left(\chi_{x}^{(l, q)}, \chi_{y}^{(l, q)}\right)$, associated with the propagation of an electron in the field $\mathbf{F}(t)$ over the time interval $\tau_{\mathrm{cl}}$ from ionization to recombination. This factor is sensitive to the spatial symmetry ( $s$ or $p$ ) of the bound state, and its $x$ and $y$ components differ significantly:

$$
\begin{gathered}
\chi_{x}^{(0,0)}=\mathcal{D} \operatorname{Ai}(\zeta), \\
\chi_{y}^{(0,0)}=\mathcal{D} \sqrt{\frac{\left|E_{0}\right|}{E}}\left[p_{y} \operatorname{Ai}(\zeta)-\mathrm{i} \frac{\varrho}{\omega_{\mathrm{at}}} \frac{\mathrm{d} p_{y}}{\mathrm{~d} t_{r}^{\mathrm{cl}}} \mathrm{Ai}^{\prime}(\zeta)\right], \\
\chi_{x}^{(1, q)}=\mathcal{D}\left[q_{j} \mathrm{Ai}(\zeta)-\mathrm{i} \frac{\varrho}{\omega_{\mathrm{at}}} \frac{\mathrm{d} q_{j}}{\mathrm{~d} t_{r}^{\mathrm{cl}}} \mathrm{Ai}^{\prime}(\zeta)\right], \\
\chi_{y}^{(1, q)}=q_{j} \chi_{y}^{(0,0)}-\mathrm{i} \mathcal{D} \sqrt{\frac{\left|E_{0}\right|}{E}} p_{y} \frac{\varrho}{\omega_{\mathrm{at}}} \frac{\mathrm{d} q_{j}}{\mathrm{~d} t_{r}^{\mathrm{cl}}} \mathrm{Ai}^{\prime}(\zeta),
\end{gathered}
$$

where $\operatorname{Ai}(\zeta)$ and $\operatorname{Ai}^{\prime}(\zeta)$ are the Airy function and its derivative, $\mathbf{p} \equiv \mathbf{p}\left(t_{r}^{\mathrm{cl}}, t_{i}\right), \mathbf{q} \equiv \mathbf{q}\left(t_{r}^{\mathrm{cl}}, t_{i}\right)$ (where the index $j$ in $q_{j}$ is $j=x$ for $q=-1$ and $j=y$ for $q=+1$ ), $t_{i}$ is given by (11), and to calculate the total derivatives of the momenta $p_{y}$ and $q_{j}$ in (15)-(17) one should use the implicit dependence of $t_{i}^{\mathrm{cl}}$ on $t_{r}^{\mathrm{cl}}$ given by equation (9). In equations (14)-(17) we also use the following definitions:

$\zeta=\varrho \frac{E-E_{\max }}{E_{\mathrm{at}}}, \quad E_{\max }=\mathcal{E}_{\max }+\Delta$,

$\Delta=\gamma_{\tau}\left[\gamma_{\tau} \mathbf{q}_{\mathrm{cl}}^{2}-\mathbf{p}_{\mathrm{cl}} \cdot \mathbf{f}\left(t_{i}^{\mathrm{cl}}\right)\right]\left(\Delta_{\mathrm{re}}+\mathrm{i} \Delta_{\mathrm{im}}\right)^{2}\left|E_{0}\right|$,

$\mathcal{D}=\frac{\varrho}{\left(v_{\mathrm{at}} t_{\mathrm{cl}}\right)^{3 / 2}} \mathrm{e}^{-\mathrm{i} \Phi_{0}}, \quad \varrho=\left(\delta \frac{I_{1}}{I_{\mathrm{at}}}\right)^{-1 / 3}$

$\delta=\frac{\gamma_{\tau}}{2}\left\{-\gamma_{\tau} \mathbf{p}_{\mathrm{cl}}^{2}+\tau_{\mathrm{cl}} \mathbf{p}_{\mathrm{cl}} \cdot \dot{\mathbf{f}}\left(t_{r}^{\mathrm{cl}}\right)+\frac{\left[\gamma_{\tau} \mathbf{p}_{\mathrm{cl}}^{2}+\mathbf{q}_{\mathrm{cl}} \cdot \mathbf{f}\left(t_{r}^{\mathrm{cl}}\right)\right]^{2}}{\gamma_{\tau} \mathbf{q}_{\mathrm{cl}}^{2}-\mathbf{p}_{\mathrm{cl}} \cdot \mathbf{f}\left(t_{i}^{\mathrm{cl}}\right)}\right\}$,

$\Phi_{0}=\left[S\left(t_{r}^{\mathrm{cl}}, t_{i}^{\mathrm{cl}}\right)+E_{0} t_{i}^{\mathrm{cl}}-E t_{r}^{\mathrm{cl}}\right] / \hbar+(3-2 l) \pi / 4, x$

where $I_{1}=c F_{1}^{2} /(8 \pi), E_{\mathrm{at}}=27.21 \mathrm{eV}, I_{\mathrm{at}}=3.51 \times$ $10^{16} \mathrm{~W} \mathrm{~cm}^{-2}$ and $\mathbf{p}_{\mathrm{cl}} \equiv \mathbf{p}\left(t_{r}^{\mathrm{cl}}, t_{i}^{\mathrm{cl}}\right)$. (Note that $\mathbf{p} \approx \mathbf{p}_{\mathrm{cl}}$ and $\mathbf{q} \approx \mathbf{q}_{\mathrm{cl}}$, as follows from (11).)

The last factor in the parametrization (12), $\hat{\mathcal{S}}_{l, q}$, is a $2 \times 2$ matrix that describes the recombination step of the $\mathrm{HHG}$ process and coincides with that in [16]. Its matrix elements do not depend on the laser parameters and involve only radial matrix elements $D_{l l^{\prime}}=\left\langle\varphi_{\kappa l}|r| \psi_{E l^{\prime}}\right\rangle$, where $\psi_{E l^{\prime}}(r)$ is the radial part of the $l^{\prime}$-wave (including the phase factor $\exp \left[\mathrm{i} \delta_{l^{\prime}}(E)\right]$ ) of the TDER scattering state $\psi_{\mathbf{P}}(\mathbf{r})\left(E=\mathbf{P}^{2} /(2 m)\right)$ [6]. The matrix elements of $\hat{\mathcal{S}}_{l, q}$ are related to the PRCS $\sigma(E, \alpha)$. For the recombination of an electron with momentum $P=\sqrt{2 m E}$ to an initially bound $\mathrm{p}$ state we have

$$
\begin{gathered}
\sigma\left(E, 0^{\circ}\right)=\frac{\hbar \Omega^{3}}{24 \pi^{2} a P c^{3}}\left|D_{10}+2 D_{12}\right|^{2}, \\
\sigma\left(E, 90^{\circ}\right)=\frac{\hbar \Omega^{3}}{24 \pi^{2} a P c^{3}}\left|D_{10}-D_{12}\right|^{2} .
\end{gathered}
$$

For recombination to an initially bound s state:

$$
\sigma_{\mathrm{s}}(E, \alpha)=\frac{\hbar \Omega^{3}}{8 \pi^{2} a P c^{3}}\left|D_{01}\right|^{2} \cos ^{2} \alpha
$$

It follows from the parametrization (12) for $\mathbf{d}_{l, q}$, that the partial rates $\mathcal{R}_{l, q}(N)$ in (4) for substates $\psi_{\mp 1}$ of a p state are different for odd $(N=2 k+1)$ and even $(N=2 k)$ harmonics and can be factorized in terms of three different atomic parameters:

$$
\begin{gathered}
\mathcal{R}_{-}^{(\mathrm{o})} \equiv \mathcal{R}_{1,-1}(2 k+1)=W^{-}(E, \phi) \sigma\left(E, 0^{\circ}\right), \\
\mathcal{R}_{-}^{(\mathrm{e})} \equiv \mathcal{R}_{1,-1}(2 k)=w^{-}(E, \phi) s_{\mathrm{at}}(E), \\
\mathcal{R}_{+}^{(\mathrm{o})} \equiv \mathcal{R}_{1,+1}(2 k+1)=w^{+}(E, \phi) s_{\mathrm{at}}(E), \\
\mathcal{R}_{+}^{(\mathrm{e})} \equiv \mathcal{R}_{1,+1}(2 k)=W^{+}(E, \phi) \sigma\left(E, 90^{\circ}\right),
\end{gathered}
$$

where the atomic parameter $s_{\text {at }}$ and the partial EWPs $W^{ \pm}$and $w^{ \pm}$are given by

$$
\begin{gathered}
s_{\text {at }}(E)=\frac{9 \hbar \Omega^{3}}{24 \pi^{2} a P c^{3}}\left|D_{12}\right|^{2}, \\
W^{ \pm}(E, \phi)=4 \pi P\left|\mathcal{T} \chi_{x}^{(1, \pm 1)}\right|^{2} / m, \\
w^{ \pm}(E, \phi)=4 \pi P\left|\mathcal{T} \chi_{y}^{(1, \pm 1)}\right|^{2} / m .
\end{gathered}
$$


The total rates for even and odd harmonics are

$$
\begin{aligned}
& \mathcal{R}^{(\mathrm{o})}=\mathcal{R}_{-}^{(\mathrm{o})}+\mathcal{R}_{+}^{(\mathrm{o})}, \\
& \mathcal{R}^{(\mathrm{e})}=\mathcal{R}_{+}^{(\mathrm{e})}+\mathcal{R}_{-}^{(\mathrm{e})} .
\end{aligned}
$$

The results (27) and (28) show clearly that the terms $\mathcal{R}_{+}^{(\mathrm{o})}$ and $\mathcal{R}_{-}^{(\mathrm{e})}$, which contain $s_{\text {at }}(E)$, in general prevent the factorization of $\mathcal{R}^{(\mathrm{o})}$ and $\mathcal{R}^{(\mathrm{e})}$ in terms of EWPs $W^{-}$and $W^{+}$and PRCSs $\sigma\left(E, 0^{\circ}\right)$ and $\sigma\left(E, 90^{\circ}\right)$. In contrast, from the parametrization (12) for $l=0$, one sees that the HHG rate $\mathcal{R}_{s}(N) \equiv \mathcal{R}_{0,0}(N)$ for an s state does factorize in terms of $\sigma_{\mathrm{s}}\left(E, 0^{\circ}\right)$ :

$$
\mathcal{R}_{\mathrm{s}}(N)=\left(W_{\mathrm{s}}^{(\mathrm{o})} \delta_{N, 2 k+1}+W_{\mathrm{s}}^{(\mathrm{e})} \delta_{N, 2 k}\right) \sigma_{\mathrm{s}}\left(E, 0^{\circ}\right),
$$

where the EWPs $W_{\mathrm{s}}^{(\mathrm{o})}(E, \phi)$ and $W_{\mathrm{s}}^{(\mathrm{e})}(E, \phi)$ for odd and even harmonics are given by equations (25) and (26) upon substituting there $\chi_{x}^{(1, \pm 1)} \rightarrow \chi_{x}^{(0,0)}$ and $\chi_{y}^{(1, \pm 1)} \rightarrow \chi_{y}^{(0,0)}$.

For both $\psi_{+1}$ and $\psi_{-1}$ substates, the comparison of HHG spectra obtained using the parametrization (12) with the exact TDER results (not shown) shows very good agreement between the two results for the dominant components $\mathcal{R}_{-}^{(\mathrm{o})}$ and $\mathcal{R}_{+}^{(\mathrm{e})}$ of the HHG yield (described by the EWPs $W^{-}$and $W^{+}$) near the cutoff of the high-energy plateau; however, for the suppressed components $\mathcal{R}_{+}^{(\mathrm{o})}$ and $\mathcal{R}_{-}^{(\mathrm{e})}$ (described by the EWPs $w^{+}$and $w^{-}$) the results using (12) are less accurate. It can be shown that the dipole moments $\mathbf{d}_{0,0}$ and $\mathbf{d}_{1,-1}$ given by equation (12) for $F_{2}=0$ coincide with those in [24] for a monochromatic field, while $\mathbf{d}_{1,+1}=0$. Since each of the three factors in the parametrization (12) has a clear physical meaning in terms of the three-step HHG scenario [25, 26] and since the propagation factor $\chi^{(l, q)}$ is insensitive to the atomic dynamics, one can extend the parametrization (12) to the case of a neutral atom by replacing the tunnelling rate $\Gamma_{\text {st }}(\tilde{F})$ and the matrix elements $D_{l l^{\prime}}$ [or $\sigma(E, \alpha)$ ] by their counterparts for a given atom, as is done in [16].

For a weak second harmonic field, a numerical analysis shows that $\left|\chi_{y}^{(1, \pm 1)}\right| \ll\left|\chi_{x}^{(1, \mp 1)}\right|$. Thus the dominant contributions to $\mathcal{R}^{(\mathrm{o})}$ and $\mathcal{R}^{(\mathrm{e})}$ are given by the first terms in equations (27) and (28). Since the second term in (27) gives a negligible contribution to $\mathcal{R}^{(\mathrm{o})}$ over a wide range of electron energies, one can approximate with high accuracy

$$
\mathcal{R}^{(\mathrm{o})} \approx W^{-}(E, \phi) \sigma\left(E, 0^{\circ}\right)
$$

Using the results (28) and (30), we compose the following ratios:

$$
\begin{gathered}
\tilde{\wp}(E, \phi)=\frac{\mathcal{R}^{(\mathrm{e})} W^{-}(E, \phi)}{\mathcal{R}^{(\mathrm{o})} W^{+}(E, \phi)}=\wp(E)+\delta(E, \phi), \\
\wp(E)=\frac{\sigma\left(E, 90^{\circ}\right)}{\sigma\left(E, 0^{\circ}\right)}, \\
\delta(E, \phi)=\frac{w^{-}(E, \phi)}{W^{+}(E, \phi)} \frac{s_{\mathrm{at}}(E)}{\sigma\left(E, 0^{\circ}\right)} .
\end{gathered}
$$

From equations (1), (30) and (32), the asymmetry parameter $\beta$ and the total PRCS $\sigma_{0}$ can be expressed in terms of $\wp$, the odd harmonic yield $\mathcal{R}^{(0)}$ and the EWP $W^{-}$:

$$
\begin{gathered}
\beta=(1-\wp) /(\wp+1 / 2), \\
\sigma_{0}=4 \pi(2 \wp+1) \mathcal{R}^{(0)} /\left(3 W^{-}\right) .
\end{gathered}
$$

Since the term $\delta(E, \phi)$ in (31) is sensitive to both the electron energy and the phase $\phi$, it is not possible in general to replace $\wp(E)$ by the phase-dependent ratio $\tilde{\rho}(E, \phi)$ for a given value of $\phi$. Equations (31)-(33) provide a way to retrieve both atomic parameters $\wp(E)$ and $s_{\text {at }} / \sigma\left(E, 0^{\circ}\right)$ by measuring the ratio of even and odd harmonic yields $\mathcal{R}^{(\mathrm{e})} / \mathcal{R}^{(\mathrm{o})}$ for two arbitrary values of the phase $\phi$. However, the retrieval procedure in this case includes the EWP $w^{-}(E, \phi)$, whose accuracy is not as good as that of $W^{ \pm}$. For this reason, below we provide a method to retrieve $\beta$ over a wide interval of energies $E$ that avoids use of the explicit form (26) for $w^{-}(E, \phi)$, by measuring the ratio $\mathcal{R}^{(\mathrm{e})} / \mathcal{R}^{(\mathrm{o})}$ for two appropriate values of $\phi$.

As an illustration, we consider HHG of Xe in a midinfrared field with parameters as in figure 1. To obtain the PRCS data, we use theoretical photoionization data [27] and the principle of detailed balance. We first choose $\phi=\phi_{0}$, for which $\left(\mathbf{p}_{\mathrm{cl}}\right)_{y}=0$. Our classical analysis shows that $\phi_{0}=\pi / 7$ (see figure 1) and this value of $\phi_{0}$ does not depend on the ratio $F_{2} / F_{1}$. Since $\mathbf{p}_{\mathrm{cl}}$ is the maximum classical momentum of the recombining electron, its direction along the polarization vector of the fundamental field gives a minimum of the ratio $w^{-}(E, \phi) / W^{+}(E, \phi)$ (see equations (20), (21), and (33)) near the cutoff ( $\left.E=E_{\text {cut }}\right)$ of the total yield of odd harmonics $\mathcal{R}^{(\mathrm{o})}$. In figures 2(a) and (b), we present our results for $\mathcal{R}^{(\mathrm{o})}$ and $\mathcal{R}^{(\mathrm{e})}$ for Xe at $\phi=\pi / 7$. In order to smooth the oscillatory pattern of HHG rates, we also present the focal-averaged rates, obtained similarly to the procedure used in [28], assuming a Gaussian distribution of laser intensity with a peak value $I_{1}=2.1 \times$ $10^{14} \mathrm{~W} \mathrm{~cm}^{-2}$. After focal averaging, we use equations (34) and (35) with $\wp$ given by $\tilde{\rho}$ in (31) to retrieve $\beta$ and $\sigma_{0}$. As shown in figures 2 (c) and (d), the retrieved results agree very well with theoretical results at the cutoff. However, the accuracy of these data significantly decreases moving to lower energies due to increasing ratio $w^{-}(E, \phi) / W^{+}(E, \phi)$ in (33).

To improve the accuracy of the retrieved values of $\sigma_{0}$ and $\beta$ over a wider interval of energies, we choose now a value for the phase $\phi=\tilde{\phi}_{0}$, for which the ratio $w^{-}(E, \phi) / W^{+}(E, \phi)$ is approximately the same over a wide interval of $E$ after focal averaging. For our field parameters, $\tilde{\phi}_{0}=\pi / 2$. (Our numerical analysis shows that this result depends only weakly on the ratio $F_{2} / F_{1}$ for $F_{2} \ll F_{1}$ and is also only slightly sensitive to $I_{1}$ and $\omega$.) Since (i) the ratio $\tilde{\rho}$ for $\phi=\phi_{0}=\pi / 7$ is most exact at the cutoff and (ii) this ratio depends only weakly on the energy $E$ for $\phi=\tilde{\phi}_{0}=\pi / 2$, we introduce the difference

$$
\Delta \tilde{\varnothing}\left(E_{\text {cut }}\right)=\tilde{\varnothing}\left(E_{\text {cut }}, \tilde{\phi}_{0}=\pi / 2\right)-\tilde{\wp}\left(E_{\text {cut }}, \phi_{0}=\pi / 7\right)
$$

for the fixed (cutoff) energy $E_{\text {cut }}$, which allows us to introduce the improved ratio $\tilde{\rho}(E)$ for $\phi=\pi / 2$ :

$$
\tilde{\wp}_{\text {imp }}(E, \pi / 2)=\tilde{\varnothing}(E, \pi / 2)-\Delta \tilde{\wp}\left(E_{\text {cut }}\right) .
$$

Figures 2(e) and (f) show that the retrieved values of $\sigma_{0}$ and $\beta$ using (34) and (35) with $\wp=\tilde{\rho}_{\text {imp }}$ agree quite well with the theoretical data over a wide energy range. Since our choice of $\phi_{0}$ and $\tilde{\phi}_{0}$ is based only on the analysis of wave packets, the suggested algorithm for retrieval of $\sigma(E, \alpha)$ is insensitive to the target atom.

In conclusion, our analytic analysis shows that the principal features of the HHG process in a two-colour laser field are its sensitive dependence on the spatial symmetry 


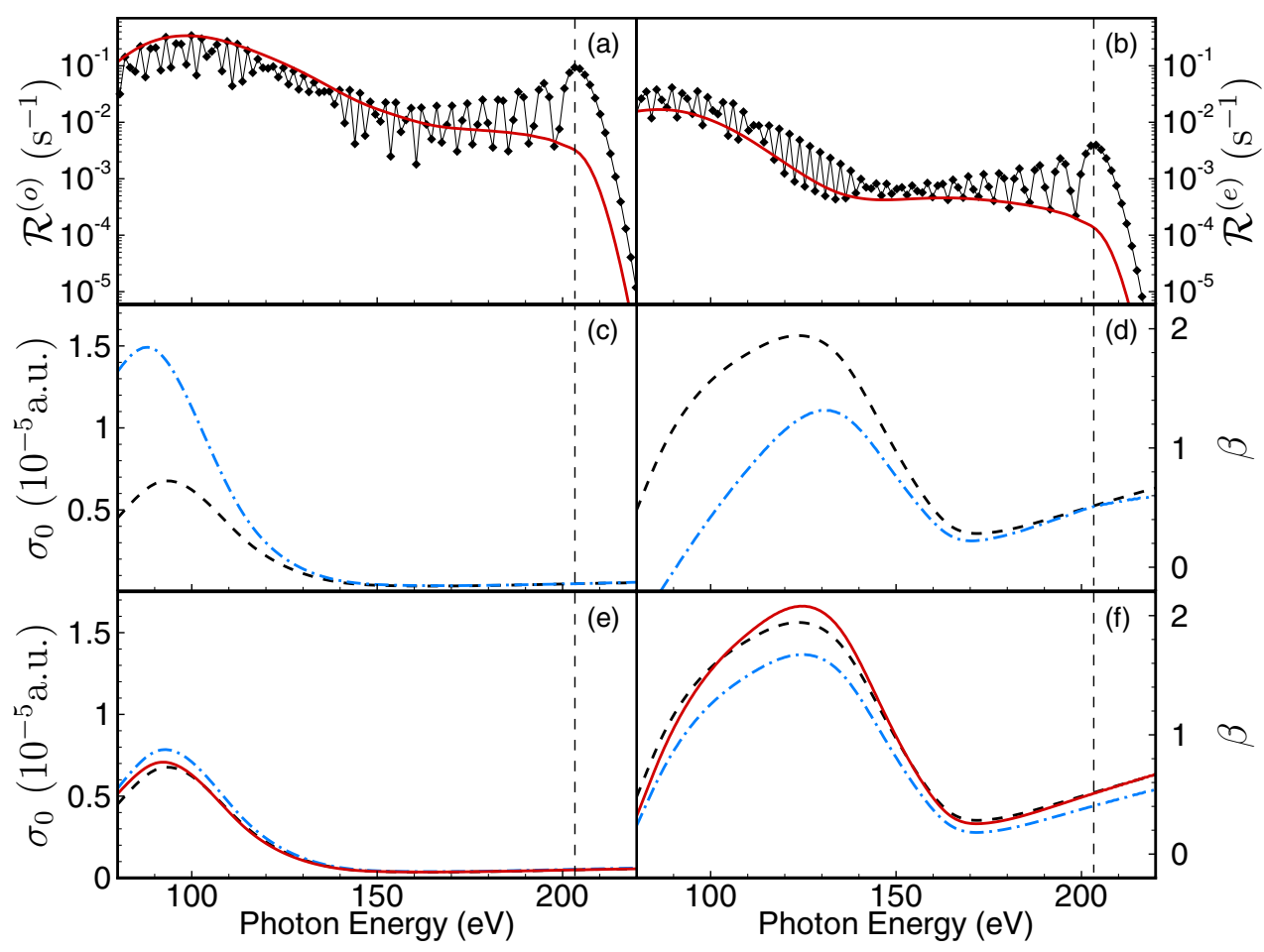

Figure 2. (a), (b) The rates $\mathcal{R}^{(\mathrm{o})}$ (a) and $\mathcal{R}^{(\mathrm{e})}$ (b) versus the harmonic photon energy $E_{\Omega}\left(=E+\left|E_{0}\right|\right)$ for HHG of Xe for laser parameters as in figure $1, \phi=\pi / 7$. Squares: results for $I_{1}=2 \times 10^{14} \mathrm{~W} \mathrm{~cm}^{-2}$ and $I_{2}=1.25 \times 10^{13} \mathrm{~W} \mathrm{~cm}^{-2}$ obtained using (27) and (28); solid (red) lines: focal-averaged results (see text) for a peak intensity $I_{1}=2.1 \times 10^{14} \mathrm{~W} \mathrm{~cm}^{-2}$. (c), (d) The total PRCS $\sigma_{0}\left(E_{\Omega}\right)(\mathrm{c})$ and the asymmetry parameter $\beta\left(E_{\Omega}\right)$ (d) for Xe. Dashed lines: theoretical results extracted from [27]; dot-dashed (blue) lines: results extracted from focal-averaged HHG spectra in (a) and (b) using (34) and (35) with $\wp=\tilde{\wp}$. (e), (f) The same as in (c), (d), but dot-dashed and solid (red) lines are results extracted from focal-averaged HHG spectra for $\phi=\pi / 2$ using (34), (35) with respectively $\wp=\tilde{\rho}$ and $\wp=\tilde{\wp} \mathrm{mp}($ see $(37))$. Laser parameters are the same as in (a), (b), but $\phi=\pi / 2$.

of the bound electron wave function and on the phase shift between the orthogonally polarized components of the field. Practical use of our analytic results for the description of twocolour HHG and the retrieval of the PRCS $\sigma(E, \alpha)$ requires only the solution of classical equations for an electron in a given two-colour field $\mathbf{F}(t)$ to obtain the ionization and recombination times $\left\{t_{i}^{\mathrm{cl}}, t_{r}^{\mathrm{cl}}\right\}$. Owing to the relative simplicity of the algorithm described in section 4 , we expect that our results should stimulate retrieval of the asymmetry parameter from experimental measurements of two-colour HHG spectra, thereby significantly extending the capability of existing methods of HHG spectroscopy with linearly polarized fields.

\section{Acknowledgments}

This work was supported in part by RFBR grant nos 12-02-12101-ofi_m and 13-02-00420-a, by NSF grant no PHY-1208059, by the Russian Federation Ministry of Education and Science (contract no 14.B37.21.1937) and by a grant from the Strategic Development Program of VSU (TSS). MYI acknowledges the warm hospitality of Voronezh State University during his collaborative visits there.

\section{References}

[1] Itatani J, Levesque J, Zeidler D, Niikura H, Pépin H, Kieffer J C, Corkum P B and Villeneuve D M 2004 Nature 432867
[2] Shiner D A, Schmidt B E, Trallero-Herrero C, Wörner H J, Patchkovskii S, Corkum P B, Kieffer J, Légaré F and Villeneuve D M 2011 Nature Phys. 7464

[3] Morishita T, Le A-T, Chen Z and Lin C D 2008 Phys. Rev. Lett. 100013903

[4] Lin C D, Le A T, Chen Z, Morishita T and Lucchese R 2010 J. Phys. B: At. Mol. Opt. Phys. 43122001

[5] Frolov M V, Manakov N L, Sarantseva T S, Emelin M Y, Ryabikin M Y and Starace A F 2009 Phys. Rev. Lett. 102243901

[6] Frolov M V, Manakov N L, Sarantseva T S and Starace A F 2011 Phys. Rev. A 83043416

[7] Frolov M V, Manakov N L, Silaev A A, Vvedenskii N V and Starace A F 2011 Phys. Rev. A 83021405

[8] Frolov M V, Manakov N L, Popov A M, Tikhonova O V, Volkova E A, Silaev A A, Vvedenskii N V and Starace A F 2012 Phys. Rev. A 85033416

[9] Okajima Y, Tolstikhin O I and Morishita T 2012 Phys. Rev. A 85063406

[10] Frolov M V, Manakov N L, Silaev A A and Vvedenskii N V 2010 Phys. Rev. A 81063407

[11] Minemoto S, Umegaki T, Oguchi Y, Morishita T, Le A T, Watanabe S and Sakai H 2008 Phys. Rev. A 78061402

[12] Wörner H J, Niikura H, Bertrand J B, Corkum P B and Villeneuve D M 2009 Phys. Rev. Lett. 102103901

[13] Cooper J and Zare R N 1969 Lectures in Theoretical Physics vol XI-C ed S Geltman, K T Mahanthappa and W E Britten (New York: Gordon and Breach) pp 317-37

[14] Shafir D, Mairesse Y, Villeneuve D M, Corkum P B and Dudovich N 2009 Nature Phys. 5412

[15] Shafir D, Soifer H, Bruner B D, Dagan M, Mairesse Y, Patchkovskii S, Ivanov M Yu, Smirnova O and Dudovich N 2012 Nature 485343 
[16] Frolov M V, Manakov N L, Sarantseva T S and Starace A F 2012 Phys. Rev. A 86063406

[17] Serbinenko V and Smirnova O 2013 J. Phys. B: At. Mol. Opt. Phys. 46171001

[18] Frolov M V, Manakov N L and Starace A F 2008 Phys. Rev. A 78063418

[19] Manakov N L, Ovsiannikov V D and Rapoport L P 1986 Phys. Rep. 141319

[20] Landau L D and Lifshitz E M 1977 Quantum Mechanics (Nonrelativistic Theory) (Oxford: Pergamon)

[21] Frolov M V, Flegel A V, Manakov N L and Starace A F 2007 Phys. Rev. A $\mathbf{7 5} 063408$

[22] Long S, Becker W and McIver J K 1995 Phys. Rev. A 522262
[23] Smirnov B M and Chibisov M I 1965 Zh. Eksp. Teor. Fiz. 49841

Smirnov B M and Chibisov M I 1966 Sov. Phys._JETP 22585

[24] Frolov M V, Manakov N L, Sarantseva T S and Starace A F 2009 J. Phys. B: At. Mol. Opt. Phys. 42035601

[25] Schafer K J, Yang B, DiMauro L F and Kulander K C 1993 Phys. Rev. Lett. 701599

[26] Corkum P B 1993 Phys. Rev. Lett. 711994

[27] Kutzner M, Radojevič V and Kelly H P 1989 Phys. Rev. A 405052

[28] Kopold R, Becker W, Kleber M and Paulus G G 2002 J. Phys. B: At. Mol. Opt. Phys. 35217 